\title{
Prevention and Treatment of Venous Thromboembolism Associated with Coronavirus Disease 2019 Infection: A Consensus Statement before Guidelines
}

\begin{abstract}
Zhenguo Zhai ${ }^{1,2,3, *}$ Chenghong Li, ${ }^{4, *}$ Yaolong Chen ${ }^{5, *}$ Grigorios Gerotziafas ${ }^{6}$ Zhenlu Zhang ${ }^{7}$ Jun Wan ${ }^{1,2,3}$ Peng Liu ${ }^{8}$ Ismail Elalamy,9,* Chen Wang1,2,3,10 On behalf of the Prevention Treatment of VTE Associated with COVID-19 Infection Consensus Statement Group, Pulmonary Embolism Pulmonary Vascular Diseases Group of the Chinese Thoracic Society, Pulmonary Embolism Pulmonary Vascular Disease Working Committee of Chinese Association of Chest Physicians, National Cooperation Group on Prevention Treatment of Pulmonary Embolism Pulmonary Vascular Disease, National Program Office for Prevention Treatment of Pulmonary Embolism Deep Vein Thrombosis, China Grade Center, Evidence-based Medicine Center of School of Basic Medical Sciences of Lanzhou University
\end{abstract}

1 Department of Pulmonary and Critical Care Medicine, Center of Respiratory Medicine, China-Japan Friendship Hospital, Beijing, China

2 Institute of Respiratory Medicine, Chinese Academy of Medical Sciences, Beijing, China

${ }^{3}$ National Clinical Research Center for Respiratory Diseases, Beijing, China

${ }^{4}$ Department of Pulmonary and Critical Care Medicine, Affiliated Hospital of Jianghan University, Wuhan, China

${ }^{5}$ Evidence-based Medicine Center, School of Basic Medical Sciences, Lanzhou University, Lanzhou, China

${ }^{6}$ Hematology and Thrombosis Center, Tenon University Hospital, INSERM UMRS 938, Sorbonne University, Paris, France

7 Department of Clinical Laboratory, Wuhan Asia Heart Hospital, Wuhan, China

${ }^{8}$ Department of Cardiovascular Surgery, China-Japan Friendship Hospital, Beijing, China

${ }^{9}$ Department of Obstetrics and Gynaecology, the First I.M. Sechenov Moscow State Medical University, Moscow, Russia

${ }^{10}$ Chinese Academy of Medical Sciences and Peking Union Medical College, Beijing, China
Address for correspondence Chen Wang, MD, PhD, Department of Pulmonary and Critical Care Medicine, China-Japan Friendship Hospital, Chinese Academy of Medical Sciences and Peking Union Medical College. No. 2 East Yinghua Road, Chaoyang District, Beijing 100029, China (e-mail: cyh-birm@263.net; zhaizhenguo2011@126.com).

Ismaïl Elalamy, MD, PhD, Hematology and Thrombosis Center, Tenon University Hospital, INSERM UMRS 938, Sorbonne University, Paris, France (e-mail: ismail.elalamy@aphp.fr).

Thromb Haemost 2020;120:937-948.
Abstract
Keywords
- COVID-19
- venous thromboembolism
- coagulation abnormalities
- prophylaxis
- consensus

Coronavirus disease 2019 (COVID-19) has caused a global pandemic in just a few months, causing millions infected. Nearly $20 \%$ of COVID-19 patients present severe coagulation abnormalities, which may occur in almost all of the severe and critical ill COVID-19 cases. Concomitant venous thromboembolism (VTE), a potential cause of unexplained deaths, has been frequently reported in COVID-19 cases, but its management is still challenging due to the complexity between antithrombotic therapy and coagulation disorders. Based on frontline practical experience and comprehensive literature review, here a panel of experts and physicians from China and Europe developed an evidence and opinion-based consensus on the prophylaxis and

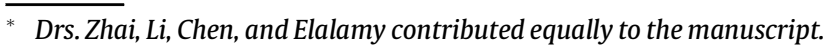

received

April 2, 2020

accepted

April 6, 2020
(C) 2020 Georg Thieme Verlag KG Stuttgart . New York
DOI https://doi.org/ $10.1055 / \mathrm{s}-0040-1710019$. ISSN 0340-6245. 
management of VTE associated with COVID-19. This statement aims for clinicians treating COVID-19 and provides practical recommendations in detailed situations, for example, how to choose thromboprophylactic measures for patients with diverse severity of disease and bleeding risk, or which kind of anticoagulant should be prescribed. With limited experience on COVID19-associated VTE, this expert consensus statement should be helpful for clinicians worldwide with specific suggestions.

\section{Key Recommendations}

1. Venous thromboembolism (VTE) risk assessment and bleeding risk assessment for coronavirus disease 2019 (COVID-19) patients

- All severe and critically ill COVID-19 patients have a high risk of VTE, so prevention of VTE is strongly recommended in absence of contraindication.

- For mild or moderate COVID-19 patients, it is recommended to determine the risk of VTE by using PADUA or IMPROVE risk assessment models (RAM) for medical patients and CAPRINI RAM for surgical patients, leading to VTE prevention in high-risk and moderate-risk patients in absence of contraindications.

- For mild or moderate COVID-19 outpatients on quarantine, it is also recommended to determine the risk of VTE using RAM for medical patients and based on the careful analysis of their clinical profile and medical history.

- Dynamic and repeated risk assessment for VTE and/or bleeding risks should be conducted for COVID-19 patients involving underlying diseases, laboratory monitoring, concomitant medications, and invasive procedures to adjust thromboprophylaxis strategy.

2. Prevention of VTE for severe or critically ill COVID-19 patients

- In severe or critically ill COVID-19 patients at high risk of bleeding or with active bleeding contraindicating temporarily pharmacological thromboprophylaxis, it is recommended to use intermittent pneumatic compression (IPC) for VTE prevention

- Pharmacological prevention with low molecular weight heparin (LMWH) is recommended as firstline treatment in patients at low or moderate risk of bleeding and with no contraindication to antithrombotic drugs. In patients with severe renal impairment (creatinine clearance rate: $<30 \mathrm{~mL} / \mathrm{min}$ ), it is recommended to use unfractionated heparin (UFH).

- In case of thrombocytopenia with suspicion of HIT, it is recommended to use nonheparin anticoagulantssuch as danaparoid, argatroban, or bivalirudin-over fondaparinux or rivaroxaban.

3. Prevention of VTE in mild and moderate COVID-19 patients

- Mild and moderate COVID-19 patients isolated for medical treatment, especially those with fever and/or gastrointestinal symptoms (diarrhea and anorexia) should be rehydrated without delay.
- Mild and moderate COVID-19 patients presenting acute medical diseases and assessed to have a high or moderate risk of VTE (PADUA or IMPROVE RAM), pharmacological prevention should be prescribed and LMWH is recommended as firstline treatment, in absence of contraindication.

- Mild and moderate COVID-19 patients requiring surgical procedure or presenting traumatic conditions and assessed to have a high or moderate risk of VTE (CAPRINI RAM), pharmacological prevention should be prescribed and $\mathrm{LMWH}$ is recommended as firstline treatment, in absence of contraindication.

- Mild and moderate COVID-19 patients perceived to have a persistent risk of VTE at the time of discharge, a prolonged outpatient VTE prophylaxis care should be considered with LMWH over DOAC use, caution due to potential drug-drug interactions and/or frequent comorbidities.

- Suspected mild and moderate COVID-19 patients should avoid sedentariness, dehydration, and should be encouraged to remain active with regular mobilization (ankle pump movements) and drinking appropriate volume of water during their isolation at home.

4. Diagnosis and treatment of VTE in COVID-19 patients

- Any change of the clinical condition should be regularly monitored in COVID-19 patients. If any corresponding VTE symptom is reported, one should be vigilant for the occurrence of DVT or pulmonary embolism (PE).

- In case of DVT or PE suspicion, diagnosis should be primarily based on careful bedside clinical examinations and then objectively confirmed by imaging explorations (venous echo-Doppler ultrasound, echocardiography, and CTPA) with mandatory clinical and protective conditions.

- In COVID-19 patients suspected for VTE, or relevant examinations fail to be conducted due to restricted conditions, starting a curative anticoagulant parenteral treatment with LMWH as firstline treatment is recommended in absence of contraindication.

- In critically COVID-19 severe cases, if there are signs of massive or high-risk PE such as hypotension or hemodynamic deterioration, in combination with the findings from bedside echocardiogram, rescue thrombolytic therapy is recommended.

- In critically COVID-19 severe cases, in refractory circulatory collapse or cardiac arrest, extracorporeal membrane oxygenation may be considered, in combination with surgical embolectomy or catheter-directed treatment. 


\section{Introduction}

Coronavirus disease 2019 (COVID-19) pneumonia is a new type of respiratory infectious disease. Since December 2019, COVID-19 patients have been found in Wuhan, Hubei Province, China. With the virus outbreak, similar cases rapidly spread across China and overseas. In clinical practice, frontline clinicians found that approximately $20 \%$ of COVID-19 patients had severe coagulation abnormalities, and almost all the patients with severe and critically ill COVID-19 infection showed major coagulation disorders. ${ }^{1,2}$ Significant abnormal coagulation parameters in severe COVID-19 patients can be prognostic suggestive. A very recent study showed that markedly elevated D-dimer and fibrinogen degradation products (FDP) were very common in COVID19-related deaths. ${ }^{3}$

Moreover, acute inflammation caused by severe infection or sepsis may affect coagulation and fibrinolysis in multiple ways, including decrease of circulating protein $C$ and antithrombin and increase of plasminogen activator inhibitor-1 (PAI-1) levels, which will finally activate coagulation cascade and inhibit fibrinolytic reaction, thus promoting thrombosis. Some COVID-19 patients, during disease progression, may suffer from sudden disease worsening, significant elevation of D-Dimer levels, and even sudden death. Therefore, more attention should be paid to the occurrence of potential pulmonary embolism (PE), following the shedding of deep venous thrombosis (DVT).

It is recommended to assess the risk of VTE, to take effective preventive measures for patients at high risk, to pay attention to VTE occurrence in asymptomatic patients or $\mathrm{PE}$ in patients with clinical manifestations of sudden deterioration of oxygenation, respiratory distress, or hypotension. So, it is important to improve awareness and to prescribe an optimal thromboprophylaxis during the prevention, control and treatment of COVID-19 infection.

In this epidemic, we organized domestic experts in the fields of evidence-based medicine, VTE prevention and treatment medicine, critical care medicine, and specially invited some experts in Wuhan jointly to draft these recommendations, which referred to the relevant diagnosis and treatment programs and clinical guidelines of COVID-19 of the National Health Commission and World Health Organization (WHO), ${ }^{4-6}$ as well as other worldwide viral infectionrelated guidelines, the prevention and management of hospitalized VTE based on evidence-based medical evidence and clinical experience. $^{7-14}$

These guidelines are applicable to help clinical frontline clinicians in risk assessment, prevention, and clinical diagnosis and VTE treatment in COVID-19 patients. However, it cannot replace the judgment of clinicians, but only strengthens the clinical management of these patients and the present unmet needs. Obviously, the prevention and control of COVID-19 infection is still the most important, but the prevention and an optimal management of vascular complications can significantly modify the prognosis and reduce the mortality.

\section{Methodology}

\section{Search Strategies}

PubMed, CBM, CNKI, Wanfang database, and guideline-relevant websites, including NICE, NGC, SIGN, WHO and GIN, etc., were retrieved. The retrieval time limited from the database construction to March 27, 2020. The searching words include "coronavirus," "middle east respiratory syndrome coronavirus," “MERS," “SARS," “2019-nCoV," “COVID-19," “WuhanCov," "Ebola virus," "Zika," "practice guideline," "recommendation" and "statement" both in Chinese and English, and VTE-relevant searching words include "venous thromboembolism," "deep vein thrombosis," "pulmonary embolism" and "pulmonary thromboembolism" both in Chinese and English. Moreover, Google academic journal preprint platforms, including MedRxiv (https://www.medrxiv.org), SSRN (https:// www.ssrn.com/index.cfm/en), ChemRxiv (https://chemrxiv. org), and BioRxiv (https://www.biorxiv.org), were retrieved manually to make a supplement for COVID-19 infection and VTE relevant practice guidelines. For clinical guidelines for which full texts were not available, we contacted the official or corresponding author by email to get full texts.

\section{Registration}

This guideline has been registered on the International Practice Guidelines Registry Platform (http://www.guidelines-registry.org) with a registration number of IPGRP2020 CN009.

\section{Inclusion and Exclusion Criteria}

Inclusion criteria: (1) practice guidelines relevant to five infectious diseases (COVID-19 infection, SARS, MERS, Ebola, and Zika) and VTE; (2) published in English or in Chinese; (3) published in peer-reviewed journals or on websites. Exclusion criteria: (1) translation or abstract of guidelines; (2) guidelines with full text not available; (3) previous versions of guidelines; and (4) bibliographic guidelines and planned guidelines.

\section{Reference Screening and Data Extraction}

The references were screened independently by two investigators (Xuan Yu and Xufei Luo) in line with the inclusion and exclusion criteria, using the reference management software (Endnote X9). During the screening process, the two investigators performed periodic crosschecking, and a third investigator would be involved in discussion for resolution in case of any disagreement. While other three investigators (Jing Wang, Meng Zhang, and Kaiyuan Zhen) performed data extraction and pairwise cross-checking independently according to the preset general information extraction table and resolved their disagreements through discussion. Extracted information includes title of the guidelines, fields covered by the guidelines, target groups, years of publication, guidelines developing organization and country, etc.

\section{Quality Assessment and Reporting}

AGREE $^{15}$ and RIGHT ${ }^{16}$ were adopted to assess methodological and reporting quality of the relevant guidelines included. 


\section{Analysis of Recommendations}

The writing group analyzed the prior guide recommendations, organized VTE seminars, and recommended the direction, intensity, and evidence quality classification, especially which had been used as a reference for the recommendations of this guidance.

\section{Part 1 Venous Thromboembolism and Bleeding Risk Assessment in Coronavirus Disease 2019 Patients}

Clinical Classification of Coronavirus Disease 2019 Infection According to the fifth edition of the diagnosis and treatment plan issued by the National Health Commission, ${ }^{4}$ the clinical classification of COVID-19 infection should meet the following criteria. (1) Mild: mild clinical symptoms, no signs of pneumonia on imaging. (2) Moderate: fever and respiratory symptoms, etc., with pneumonia signs on imaging. (3) Severe: patients with any of the following conditions: respiratory distress with respiratory rate $\geq 30$ breaths/min; $\mathrm{SPO} 2 \leq 93 \%$ at rest; $\mathrm{PaO} 2$ / FiO2 $\leq 300 \mathrm{~mm} \mathrm{Hg}(1 \mathrm{~mm} \mathrm{Hg}=0.133 \mathrm{kPa})$. (4) Critically ill: patients with any of the following conditions: respiratory failure requiring mechanical ventilation; shock; other organ failure requiring admission to Intensive Care Unit (ICU).

\section{Risk Factors and Risk Assessment for Venous Thromboembolism}

Based on the current publications, most patients with COVID-19 infection may have fever ${ }^{1,2}$ and dehydration due to physical cooling or drug administration. In addition, quite a few patients may have gastrointestinal complication in later period, including diarrhea or anorexia which may lead to serious nondominant and dominant dehydration, insufficient fluid volume, hemoconcentration, and so on, which lead to blood viscosity increase. Dehydration is also a wellknown VTE risk factor.

COVID-19 patients may have many VTE risk factors once severe patients combined with other infections (e.g., bacteria, fungi, etc.), bedridden, obesity, or other comorbidities, especially in the elderly and people with primary diseases, ${ }^{2}$ with increased occurrence of hypotension, shock, coma, or sedation ICU patients that may lead to slower limb venous blood flow and venous stasis.

The release of a large amount of inflammatory mediators and the application of hormones and immunoglobulin in severe or critically ill patients may lead to a blood hypercoagulability. Furthermore, mechanical ventilation, central venous catheterization, and surgery may cause vascular endothelial injury. The combination of all the above factors may lead to DVT occurrence or even the possibility of lethal PE due to thrombus migration.

Therefore, VTE risk assessment scoring should be evaluated for all COVID-19 in-patients. It is recommended to evaluate VTE risk based on many different clinical conditions ${ }^{7,8,17,18}$ :

1. Age $\geq 40$ years, bedridden $\geq 3$ days, confirmed with COVID-19 infection and combined with one of the following diseases or risk factors (age $\geq 75$ years, acute infectious disease [especially severe infection or sepsis], respiratory failure, heart failure [New York Heart Association Class III or IV], obesity [body mass index: $\geq 30 \mathrm{~kg} / \mathrm{m}^{2}$ ], previous history of VTE, acute exacerbation of chronic obstructive pulmonary disease, acute cerebral infarction, acute coronary syndrome, lower limbs varicose veins, malignant tumors, inflammatory bowel disease, and chronic kidney disease) which are considered to lead to a high risk of VTE.

2. It is recommended to use PADUA VTE risk assessment model (RAM) or IMPROVE VTE RAM for internal medicine department COVID-19 patients. ${ }^{8,17}$

3. It is recommended to use CAPRINI RAM for surgical operation or trauma patients suspected or confirmed with COVID-19 infection. ${ }^{18}$

4. Pregnant or postpartum COVID-19 patients are also at higher VTE risk with factors such as age over 35 years old, history of VTE, preeclampsia, intrauterine growth retardation, genetic thrombophilia, blood transfusion, postpartum infection, systemic lupus erythematosus, heart disease or sickle-cell anemia, obesity, multiple pregnancies, postpartum hemorrhage, etc. ${ }^{19,20}$

5. Due to epidemic spread, many hospitals are now so full that many patients with mild or even moderate COVID-19 are sent home on quarantine. It is still recommended to identify possible candidates for thromboprophylaxis among these "ambulatory" patients based on their clinical profiles (overweight, comorbidities, cardiovascular risk factors, immobilization, etc) and their clinical history (previous DVT, previous arterial thrombosis, familial thrombophilia, etc).

6. COVID-19 infection is often associated with a severe inflammation leading to a major blood hypercoagulability with a high risk of DVT. Therefore, various coagulation parameters abnormalities can be markers of this thrombotic risk such as an abrupt increase of D-dimer level or PAI-1 rates, or a rapid decrease of protein C or antithrombin levels ${ }^{1-3}$. In case of any clinical symptom evoking VTE episode or a worsening of coagulation parameters such as a D-dimer elevation, imaging examination should be performed to rule out or confirm thrombosis.

\section{Bleeding Risk Assessment in Coronavirus 2019 Patients} Bleeding-relevant risk factors include the following aspects $^{7,8,10}$ :

1. Patient factors:
(a) Age $\geq 85$ years
(b) Previous bleeding episode
(c) Coagulation factors disorders
(d) Platelet count $<50 \times 10^{9} / \mathrm{L}$
(e) Constitutive hemorrhagic disorder (Willebrand dis- ease, platelet dysfunction, etc.)

2. Underlying diseases:

(a) Active bleeding, such as uncontrolled peptic ulcer

(b) Uncontrolled hypertension (systolic pressure $>180 \mathrm{~mm} \mathrm{Hg}$ and/or diastolic pressure $>110 \mathrm{~mm} \mathrm{Hg}$ )

(c) Intracranial diseases that may lead to severe hemorrhage, such as acute stroke (within 3 months), serious cerebral or acute spinal cord injury, or acute spinal cord injury 

(d) Diabetes
(e) Malignant tumor
(f) Renal impairment or hepatic failure, etc

3. Concomitant medications: anticoagulant drugs, antiplatelet drugs, or thrombolytic drugs are currently used

4. Invasive procedure: 4 hours before and 12 hours after receiving surgery, lumbar puncture, and epidural anesthesia

The influence of bleeding risks on antithrombotic preventive strategies, treatment protocols, drugs, and dose selection should be taken into full consideration in patients meeting any of the aforementioned factors.

\section{Recommendations for Venous Thromboembolism Risk Assessment and Bleeding Risk Assessment in Coronavirus Disease 2019 Patients}

- All severe and critically ill COVID-19 patients have a high risk of VTE, so prevention of VTE is strongly recommended in absence of contraindication.

- For mild or moderate COVID-19 patients, it is recommended to determine the risk of VTE by using PADUA or IMPROVE risk assessment models (RAM) for medical patients and CAPRINI RAM for surgical patients, leading to VTE prevention in high-risk and moderate-risk patients in absence of contraindications.

- For mild or moderate COVID-19 outpatients on quarantine, it is also recommended to determine the risk of VTE using RAM for medical patients and based on the careful analysis of their clinical profile and medical history.

- Dynamic and repeated risk assessment for VTE and/or bleeding risks should be conducted for COVID-19 patients involving underlying diseases, laboratory monitoring, concomitant medications, and invasive procedures to adjust thromboprophylaxis strategy.

Part 2 Prevention of Venous Thromboembolism In Severe and Critically III Coronavirus Disease 2019 Patients

During the treatment of COVID-19 patients, appropriate thromboprophylactic strategies should be developed according to VTE and bleeding risk assessment combined with patients' clinical conditions and taking a dynamic view.

Preventive Strategies for Severe and Critically Ill Patients at Low Bleeding Risk

For severe and critically ill COVID-19 patients at low bleeding risk, subcutaneous injection of low molecular weight heparin (LMWH) is recommended as firstline treatment for prevention. ${ }^{7,8,12,13}$ As the panels of experts suggest in critically ill medical patients, using LMWH over UFH, most of patients can receive a dose of 4,000 IU of enoxaparin or $2,850 \mathrm{IU}$ of nadroparin once daily. The dose should be adjusted based on the specific condition of the patient: overweight 90 to $130 \mathrm{~kg} \mathrm{6,000} \mathrm{IU/d}$ and obese 4,000 $\mathrm{IU} \times 2$ / $\mathrm{d}$ of enoxaparin as in bariatric surgery for example.

As LMWH is mainly eliminated by renal route, attention should be paid to the patient's kidney function. Patients with renal impairment should use LMWH with caution. If LMWH is administered in patients with renal impairment, dose adjustment should be considered according to the monitoring of anti-Xa activities. Patients with severe renal dysfunction can receive subcutaneous injection of UFH at a dose of $5,000 \mathrm{U}$ twice-daily. ${ }^{21,22}$ The dose must be also adjusted based on the specific condition of the patient in clinical practice. In intensive care units (ICU), with critically ill COVID-19 patients, it is recommended to adopt such a drug prevention associated with or without mechanical prevention. For patients under extracorporeal membrane oxygenation (ECMO) support with heparin, the dose regimen is already curative with a careful monitoring. ${ }^{23}$

The administration of heparin drugs may lead to heparininduced thrombocytopenia (HIT). A careful monitoring should be implemented and 4T's scoring performed to think about HIT. ${ }^{24}$ In patients presenting a significantly relative thrombocytopenia and suspected to develop HIT during heparin administration, it is recommended to use nonheparin anticoagulant drugs such as danaparoid, argatroban, or bivalirudin. ${ }^{24}$ Considering all comorbidities, patient frailty, and potential drug-drug interactions, others nonheparin drug use, such as rivaroxaban or fondaparinux, are quite problematic in this complex clinically instable context. ${ }^{25,26}$

During the prevention of VTE, bleeding or coagulation abnormalities may occur following the administration of anticoagulant drugs. Once it happens, drug administration should be stopped immediately, and appropriate actions can be taken. Moreover, preventive means should be adjusted dynamically based on the change of bleeding risks. Drug prevention should be conducted throughout the whole period of ICU admission or until the risk factors are removed.

Bleeding risk seems much less important than thrombotic risk in such a context. The physical methods only should be not enough to control this clinical hypercoagulability. DIC occurrence is more common in this situation, with a resistance to thromboprophylaxis in that acute situation. The classical thromboprophylaxis dosage may be not enough for this condition. Therefore, a careful monitoring is mandatory with anti-Xa in case of UFH treatment due to numerous interactions with inflammatory proteins reducing its bioavailability and interferences with aPTT prolongation, which is less predictable of the anticoagulation activity. ${ }^{27,28}$

Thromboprophylaxis is required in all ICU patients, choosing mainly LMWH with longer polysaccharide chains less eliminated by kidney route such as tinzaparin or dalteparin, which can be used even in cases of creatinine clearance between 20 and $30 \mathrm{~mL} / \mathrm{min}^{28-30}$ Nevertheless, VTE can occur despite well-conducted thromboprophylaxis. ${ }^{31}$

\section{Prevention Strategies for Severe and Critically Ill Patients at High Risk of Bleeding}

Most severe and critically ill COVID-19 patients may have underlying diseases, coagulation disorders, hormone treatment history, or other circumstances such as severe liver dysfunction, resulting the increase of bleeding risk, so factors that may lead to bleeding should be corrected actively.

No study has prospectively evaluated the efficacy and safety of DVT prophylaxis in critically ill patients. Thus, the 
use of pharmacological prophylaxis in these patients should be carefully balanced against the risk of bleeding. 8,31

Mechanical prevention such as intermittent pneumatic compression (IPC) and graduated compression stockings (GCS) should be conducted. Mechanical prevention should be conducted throughout the whole period of ICU admission or until major bleeding risk factors are removed. Among critically ill patients who receive pharmacologic thromboprophylaxis, adjunctive IPC cannot result in a significantly lower incidence of proximal lower-limb DVT than pharmacologic thromboprophylaxis alone. ${ }^{32}$ Therefore, once the bleeding risk decreases, the pharmacologic thromboprophylaxis should be resumed as earlier as possible.

If IPC will be used, medical staff should be trained for its correctly use. It needs to be mentioned that GCS has limited preventive value for ICU patients who are absolutely bedridden, and it is often used as an auxiliary means to IPC. Patients at high risk of bleeding are recommended to use IPC and (or) in combination with GCS until the risk of bleeding is decreased. If patients leave ICU and start up activities, but the activities are limited, and other risk factors of VTE still exist, the use of GCS can be considered.

Prior to the use of GCS, patients should be guided by professionally trained medical staff to avoid adverse reactions induced by improper use of GCS. In case of any adverse reaction, occurrence of complications can be avoided by changing the size and the materials, applying emollient and reducing the degree of compression. Moreover, patients who need to use GCS or their family members should be educated to ensure the standardized application of GCS.

\section{Recommendations for Prevention of Venous Thromboembolism in Severe and Critically III Coronavirus Disease 2019 Patients}

- In severe or critically ill COVID-19 patients at high risk of bleeding or with active bleeding contraindicating temporarily pharmacological thromboprophylaxis, it is recommended to use IPC for VTE prevention.

- Pharmacological prevention with LMWH is recommended as firstline treatment in patients at low or moderate risk of bleeding and with no contraindication to antithrombotic drugs. In patients with severe renal impairment (creatinine clearance rate: $<30 \mathrm{~mL} / \mathrm{min}$ ), it is recommended to use unfractionated heparin (UFH).

- In case of thrombocytopenia with suspicion of HIT, it is recommended to use nonheparin anticoagulants-such as danaparoid, argatroban, or bivalirudin-over fondaparinux or rivaroxaban.

\section{Part 3 Prevention of Venous Thromboembolism in Mild and Moderate Coronavirus Disease 2019 Patients}

Treatment in isolation is an effective means to avoid the transmission route for patients with mild and moderate COVID-19 infection. Treatment in isolation makes the space for restricted activities, reduced time for activities, and increased time of sedentariness or bed rest, which will slow down the venous blood flow in the lower limbs. Venous blood stasis is prone to cause DVT in the lower limbs. Judging from the current received cases, most mild or moderate COVID-19 patients have a good prognosis. Therefore, for mild or moderate COVID-19 patients, attention should be paid to the change and to the occurrence of adverse events based on active isolation and general treatment. If there is no other risk, general preventive measures can be given to these patients. In general, it is recommended for the patients to drink more water and do appropriate activities in the isolated areas. If mild or moderate COVID-19 patients with internal medicine diseases or surgical diseases are evaluated to have high-risk or moderate risk of VTE with PADUA or CAPRINI RAMs and have no anticoagulant contraindications, thromboprophylaxis should be considered, and LMWH should be recommended as firstline treatment. The duration of drug prevention should be at least 7 to 10 days in principle or until vascular risk factors removed.

Patients with fever or gastrointestinal symptoms (e.g., diarrhea and anorexia) may suffer from invisible and visible dehydration. It is recommended to carry out health education on patients in terms of prevention of VTE and ask patients to drink regularly more water. At the time of bed rest, patients should be encouraged to do some bedside movement or guided to engage in in-bed active or passive movement such as ankle pump movements. If the patient has difficulty in drinking water, suffers from serious dehydration, and has the risk of electrolyte disturbance, intravenous fluid and electrolytes supplementation must be conducted.

Discharged patients may still have the risk of VTE due to less activity during hospitalization or due to the presence of other complications, so it is needed to assess whether the patient has VTE or whether the patient still has VTE risk factors after discharge. If the patient is still at high risk of VTE on discharge, continuing the preventive measures such as subcutaneous injection of LMWH can be considered outside the hospital with a prolonged thromboprophylaxis over DOACs use. ${ }^{8}$ Apart from reasons related to local approval status, an important reason to be cautious with DOACs would be their possible interactions with some experimental treatments against severe acute respiratory syndrome coronavirus 2 (SARS-CoV-2). Most of these antiviral agents like lopinavir/ritonavir may increase the DOAC-related bleeding risk via CYP3A4 and/or P-gp inhibition. Moreover, potential organ dysfunction with a significant transaminase increase or a compromised creatinine clearance could also limit the DOACs prescription in these fragile recovering patients.

If pregnant COVID-19 women with increased risk of VTE have contraindications to pharmacologic thromboprophylaxis, mechanical prevention (GCS or IPC) is recommended. In women after urgent cesarean section with a higher risk of VTE or presenting associated risk factors of VTE after delivery, it is recommended to maintain pharmacologic thromboprophylaxis and/or mechanical prevention with stockings. The duration of such a thromboprophylaxis can be lengthened to 6 to 8 weeks after pregnancy or until VTE risk factors removed.

Patients who have been discharged and stayed in the isolated areas (such as square cabin hospitals) should be asked to do appropriate activities: drink more water for 
regular hydration and to avoid sedentariness or long-term bed rest.

In case of any VTE clinical presentations such as swelling, pain, bruising, redness with unilateral, or bilateral lower extremities; chest tightness, chest pain, or dyspnea, objective confirmation of VTE is needed. If these occur, refrain from massaging the affected limbs or engaging in strenuous activities, patient should inform the competent physician as soon as possible for assessment and proceed to the nearest hospital for imaging and treatment.

\section{Recommendations for Prevention of Venous Thromboembolism in Mild and Moderate Coronavirus Disease 2019 Patients}

- Mild and moderate COVID-19 patients isolated for medical treatment, especially those with fever and/or gastrointestinal symptoms (diarrhea and anorexia) should be rehydrated without delay.

- Mild and moderate COVID-19 patients presenting acute medical diseases and assessed to have a high or moderate risk of VTE (PADUA or IMPROVE RAM), pharmacological prevention should be prescribed and LMWH is recommended as firstline treatment, in absence of contraindication.

- Mild and moderate COVID-19 patients requiring surgical procedure or presenting traumatic conditions and assessed to have a high or moderate risk of VTE (CAPRINI RAM), pharmacological prevention should be prescribed and LMWH is recommended as firstline treatment, in absence of contraindication.

- Mild and moderate COVID-19 patients perceived to have a persistent risk of VTE at the time of discharge, a prolonged out-patient VTE prophylaxis care should be considered with LMWH over DOAC use, caution due to potential drugdrug-interactions, and/or frequent comorbidities.

- Suspected mild and moderate COVID-19 patients should avoid sedentariness, dehydration, and should be encouraged to remain active with regular mobilization (ankle pump movements) and drinking appropriate volume of water during their isolation at home.

\section{Part 4 Diagnosis and Treatment of Venous}

Thromboembolism in Coronavirus Disease 2019 Patients Dynamic monitoring on the change of their condition is required for moderate, severe, or critically ill or discharged patients. Especially for patients who are bedridden for more than 3 days, if they present asymmetrical pain, swelling, or discomfort of unilateral or bilateral lower limbs, or local swelling of extremities or superficial venous filling in case of central vein catheterization, it is necessary to document the occurrence of DVT by venous echo-Doppler ultrasound. In case of chest pain, hemoptysis, dyspnea, and hypoxemia aggravation, we should suspect and confirm the occurrence of PE by computed tomography pulmonary angiography (CTPA). We also recommend using bedside echocardiography for initial assessment of such patients, especially in the emergency department because it is cheap, convenient, and safe, particularly in the COVID-19 setting. Current ESC guidelines also emphasize it for suspected (severe) PE, especially in unstable patients. ${ }^{14}$ All the physicians must be aware that VTE can be often asymptomatic in COVID-19 patients.

\section{Evaluation of Coagulation Function and D-Dimer Testing}

The dynamic change in D-dimer or other coagulation indicators should be monitored in hospitalized COVID-19 patients if available. The increase of D-dimer in the early stage of pneumonia is logical with the inflammatory response, but a sudden and rapid rise of D-dimer rate with respiratory failure manifestation often indicates an acute increase of inflammatory response and a worsening progress of the disease. When the condition is controlled, D-dimer levels may gradually decrease and return to subnormal rates. D-dimer levels are also elevated in plasma in case of acute thrombosis with simultaneous activation of both coagulation and fibrinolysis. Multiorgan dysfunctions and systemic coagulopathy are reported to be associated with a high mortality rate in patients with COVID-19 infection.

There are some clinical studies having been conducted in Wuhan, Hubei province which is the center of COVID-19 outbreak since January 2020, evaluating the coagulation parameters of COVID-19 cases, and showing that they may have prognostic values and be important therapeutic markers. $^{3,33}$ A prospective cohort study in hospitalized patients with confirmed COVID-19 infection reported the significant increase of D-dimer levels and FPDs, as well as the prolongation of PT and aPTT in nonsurvivors compared with those parameters in survivors on admission $(p<0.05)$. Moreover, $71.4 \%$ of nonsurvivors and $0.6 \%$ survivors had overt disseminated intravascular coagulation (DIC) during their hospital stay. These data suggest that an important hypercoagulable profile and an overt DIC are associated with a poor prognosis and an increase of deaths in COVID-19 patients. $^{3}$ Another prospective cohort of hospitalized patients with confirmed SARS CoV-2 infection, reported that antithrombin rates were significantly decreased compared with a healthy control group $(p<0.001)$. The values of D-dimer, FDP, and fibrinogen in all SARS-CoV-2 patients were substantially higher than those of healthy controls, suggesting that monitoring of D-dimer and FDP values may be helpful for the early identification of severe cases. Heparin treatment appears to be associated with better prognosis in severe COVID-19 patients with coagulopathy. ${ }^{33}$ Therefore, coagulation disturbances are related to a disease aggravation and a poor clinical prognosis in patients with COVID-19 infection. $^{34}$ In a retrospective, multicenter cohort study, multivariable regression analysis showed that in adult inpatients with COVID-19 infection, D-dimer greater than $1 \mathrm{mg} / \mathrm{L}$ and several other clinical risk factors including elderly and higher sequential organ failure assessment (SOFA) score were correlated with the in-hospital death. ${ }^{35}$ Another retrospective cohort study of 201 hospitalized patients with confirmed COVID-19 pneumonia showed also that organ and coagulation dysfunctions were associated with ARDS occurrence and the worse disease progression from ARDS to death. $^{36}$ 
In COVID-19 patients, it seems that coagulation parameters with prognostic values, such as D-dimer and FDP increase, can be important therapeutic markers. Physicians can follow their evolution to determine not only the severity and the progression of illness but also to determine the risk of developing VTE, and therefore, the need to increase heparin dose from prophylactic to curative regimen. If COVID-19 infection is controlled and stable whereas D-dimer levels are rapidly increasing or such a D-dimer significant increase appears without evidence of the underlying disease progress, venous echo-doppler ultrasound of bilateral lower extremities should be conducted to rule out or confirm DVT.

In COVID-19 patients, coagulation disturbances are related to both disease aggravation and poor clinical prognosis. Therefore, monitoring of D-dimer, fibrinogen and FDP values may be helpful for the early identification of a thrombotic complication or a worsening progress of the disease with overt DIC leading thus to a careful reevaluation of the therapeutic strategy.

\section{Diagnosis and Treatment of Deep Venous Thrombosis}

In case of DVT suspicion, diagnosis should be primarily based on careful bedside clinical examinations and then objectively confirmed by imaging explorations (venous echo-Doppler ultrasound). In COVID-19 patients, suspicion for VTE should be related to unexplained leg swelling or pain associated with an important and unexpected increase of D-dimer. The most commonly used RAM, Wells score, might not be so helpful in this context, with a majority of patients at intermediate or high probability of DVT and high level of Ddimers. The other limitations of the Wells score are its complexity, its subjectivity, and the possible interaction of personal interpretation of each criteria. Furthermore, to exclude or confirm any DVT episode lower extremity echoDoppler ultrasound is not always easy to perform due to patient positioning in ICU.

So, in case of suspicion or incidental DVT, a classical therapeutic anticoagulation must be started. LMWH is again the firstline treatment with a dose based on the patient body weight (e.g., enoxaparin $100 \mathrm{IU} / \mathrm{kg}$, twice daily s.c., or enoxaparin $150 \mathrm{IU} / \mathrm{kg}$, once daily, s.c. or nadroparin, $86 \mathrm{IU} / \mathrm{kg}$ twice daily, s.c.). In case of severe renal impairment, UFH is preferable with a careful monitoring based on Anti-Xa activity (target $0.3-0.7 \mathrm{UI} / \mathrm{mL}$ ) and regular platelet count to control HIT occurrence. VKA and DOACs are difficult to propose in this acute situation with many limitations related to drug-drug interactions and potential comorbidities with renal or liver dysfunction. This antithrombotic oral option should be discussed case by case depending on the patient preferences, evolution, and treatment.

\section{Diagnosis and Treatment of Pulmonary Embolism}

Any change of clinical condition should be regularly monitored in COVID-19 patients. If any corresponding VTE symptom is reported, one should be vigilant for the occurrence of PE. In case of PE suspicion, diagnosis should be primarily based on careful bedside clinical examinations and then objectively confirmed by imaging explorations (venous echo-Doppler ultrasound, echocardiography and CTPA) with mandatory clinical and protective conditions. If there is evidence of DVT in the lower extremities and/or evidence of right-ventricular thrombus, the patient must be treated as an authentic acute PE. If there is no DVT evidence, isolated PE remains possible and CTPA is recommended to exclude PE if protective conditions are available. Considering the transmission risk of COVID-19 infection and the false positive factors induced by lung lesions, it is not recommended to base PE diagnosis on pulmonary ventilation-perfusion imaging.

Details are given in the Guidelines on Diagnosis, Treatment, and Prevention of Pulmonary Thromboembolism ${ }^{9}$ and Guidelines on the Prevention and Treatment of Thrombotic Diseases in China. ${ }^{10}$ However, considering the infectivity and other characteristics of COVID-19, the identification and treatment of PE need to be adjusted according to the actual condition. If the disease is severe or due to limited conditions that cannot allow a relevant examination, if there is no anticoagulant contraindication, it is recommended to start curative anticoagulant therapy. The firstline treatment is based on parenteral anticoagulation with LMWH in absence of contraindication (e.g., enoxaparin $100 \mathrm{IU} / \mathrm{kg}$, twice daily s. c., or enoxaparin $150 \mathrm{IU} / \mathrm{kg}$, once daily, s.c. or nadroparin, $86 \mathrm{IU} / \mathrm{kg}$ twice daily, s.c.). In case of severe renal impairment, UFH is proposed by venous infusion and then by subcutaneous route with a regular monitoring as mentioned above for anticoagulation dose adjustment frequently required in this inflammatory context. The DOACs are an option only after the acute phase if the patient is more stable with no more treatment interfering with CYP3A4 and P-gp pathways and no more major comorbidities.

During the diagnosis and treatment of COVID-19 infection, if the condition suddenly worsens and there are signs of massive or high-risk PE such as hypotension or sudden cardiac arrest, and that bedside echocardiography implies new onset of increased right-ventricular load or pulmonary arterial hypertension, which fail to be explained with primary pneumonia, thrombolytic therapy should be urgently initiated. $^{12-14}$ In critically COVID-19 severe cases, in refractory circulatory collapse or cardiac arrest, ECMO may be considered, in combination with surgical embolectomy or catheter-directed treatment, as rescue initiatives. ${ }^{12-14}$

\section{Recommendations for Diagnosis and Treatment of Venous Thromboembolism in Coronavirus Disease 2019 Patients}

- Any change of the clinical condition should be regularly monitored in COVID-19 patients. If any corresponding VTE symptom is reported, one should be vigilant for the occurrence of DVT or PE.

- In case of DVT or PE suspicion, diagnosis should be primarily based on careful bedside clinical examinations and then objectively confirmed by imaging explorations (venous echo-Doppler ultrasound, echocardiography, and CTPA) mandatory clinical and protective conditions.

- In COVID-19 patients suspected for VTE, or relevant examinations fail to be conducted due to restricted 
conditions, starting a curative anticoagulant parenteral treatment with LMWH as firstline treatment is recommended in absence of contraindication.

- In critically COVID-19 severe cases, if there are signs of massive or high-risk PE such as hypotension or hemodynamic deterioration, in combination with the findings from bedside echocardiogram, rescue thrombolytic therapy is recommended.

- In critically COVID-19 severe cases, in refractory circulatory collapse or cardiac arrest, ECMO may be considered, in combination with surgical embolectomy or catheterdirected treatment.

\section{Conclusion}

Given the specificity and complexity of COVID-19, isolation, protection, and supportive antithrombotic treatment are the main challenges for frontline clinicians. As more and more knowledge and experience on the COVID-19 accumulated over time, therapy for this disease will become mature. $^{37,38}$ However, some potential complications may lead to poor prognosis. Those with COVID-19 and preexisting cardiovascular disease have an increased risk of severe disease and death. Infection has been associated with multiple direct and indirect cardiovascular complications including acute myocardial injury, myocarditis, arrhythmias, and VTE. 39

Both thrombotic risk assessment and VTE prevention are important components of the complex and comprehensive treatment of COVID-19 infection. The fact that some patients' conditions may change rapidly could result in dynamic modifications of thrombotic risk and bleeding during treatment. Thus, repeated assessment and optimized strategies are necessary to reduce the occurrence of VTE and prevent fatal PE incidences, and effectively ensure the safety of patients and promote early recovery.

These guidelines should contribute to improve thromboprophylaxis and treatment in COVID-19 patients and their clinical outcomes. Finally, we would like to pay tribute to all the health care professionals who are battling on the front line of the COVID-19 epidemic.

\section{Expert Group Members}

From Pulmonary Embolism and Pulmonary Vascular Diseases Group of the Chinese Thoracic Society, Pulmonary Embolism and Pulmonary Vascular Disease Working Committee of Chinese Association of Chest Physicians, National Cooperation Group on Prevention and Treatment of Pulmonary Embolism and Pulmonary Vascular Disease, National Capacity Building Program Office for Prevention and Treatment of Pulmonary Embolism and Deep Vein Thrombosis, China Grade Center, Evidence-based Medicine Center of School of Basic Medical Sciences of Lanzhou University.

Reviewed by

Chinese Academy of Medical Sciences and Peking Union Medical College (Chen Wang) and Department of Cardio- vascular Surgery and Vascular Medicine of China-Japan Friendship Hospital (Peng Liu).

\section{Drafted by}

Evidence-based Medicine Center of Lanzhou University (Yaolong Chen); China-Japan Friendship Hospital (Jun Wan, Wanmu Xie, and Zhenguo Zhai); Affiliated Hospital of Jianghan University (Chenghong Li); Tongji Hospital Affiliated to Tongji Medical College of Huazhong University of Science and Technology (Xiansheng Liu); the First Affiliated Hospital of Chongqing Medical University (Hong Chen); and the First Affiliated Hospital of Dalian Medical University (Yingqun Ji).

\section{Wuhan Frontline Antiepidemic Experts}

General Hospital of Northern Theater Command (Zhuang $\mathrm{Ma}$ ); Beijing Hospital (Xiaomao Xu); Huashan Hospital Affiliated to Fudan University (Shengqing Li); the Second Hospital of Hebei Medical University (Yadong Yuan); Tongji Hospital Affiliated to Tongji Medical College of Huazhong University of Science and Technology (Xiansheng Liu and Jianping Zhao); Affiliated Hospital of Jianghan University (Chenghong Li and Ziyang Zhu); the First Affiliated Hospital with Nanjing Medical University (Xuesong Chen); Renmin Hospital of Wuhan University (Ke $\mathrm{Hu}$ ); Wuhan Asia Heart Hospital (Zhenlu Zhang), Wuhan Pulmonary Hospital (Ronghui Du); Wuhan Jinyintan Hospital (Ming Wei); the Central Hospital of Wuhan (Yi Hu); the First Affiliated Hospital of Zhengzhou University (Zhe Cheng); and China-Japan Friendship Hospital (Shuai Zhang).

\section{Discussed by}

Peking University International Hospital (Shuang Liu); Peking Union Medical College Hospital (Juhong Shi); the First Affiliated Hospital of Chongqing Medical University (Hong Chen); the First Affiliated Hospital of Dalian Medical University (Yingqun Ji); the First Affiliated Hospital of Guangzhou Medical University (Chunli Liu); Tongji Hospital Affiliated to Tongji Medical College of Huazhong University of Science and Technology (Xiansheng Liu); the Second Hospital of Jilin University (Chunling Dong); the First Medical Center of Chinese PLA General Hospital (Weihua Zhang); Tangdu Hospital of Air Force Military Medical University (Ning Wang); Affiliated Hospital of Nantong University (Songshi Ni); the Affiliated Hospital of Qingdao University (Zhaozhong Cheng); Shandong Provincial Hospital (Ling Zhu); Ruijin Hospital Affiliated to Shanghai Jiao Tong University (Guochao Shi); Central Hospital Affiliated to Shenyang Medical College (Shuyue Xia); Shenzhen People's Hospital (Yingyun Fu); Beijing Chao-yang Hospital of Capital Medical University (Yuanhua Yang); West China Hospital of Sichuan University (Qun Yi); Shanghai Pulmonary Hospital Affiliated toTongji University (Jinming Liu); the First Affiliated Hospital of Zhengzhou University (Jianjun Jin); Fuwai Hospital of CAMS and PUMC (Changming Xiong and Zhihong Liu); Xiangya Hospital of Central South University (Yongjun 
Tang); and China-Japan Friendship Hospital (Jun Wan, Wanmu Xie, Qian Gao, Yihong Sun, and Zhenguo Zhai).

Evidence Evaluated by

Beijing Hospital (Jing Wang); the First Affiliated Hospital of Chongqing Medical University (Yuliang Liu and Xiaohui Wang); Affiliated Hospital of Jianghan University (Qin Wei); Evidence-based Medicine Center of School of Basic Medical Sciences of Lanzhou University (Xuan Yu and Xufei Luo); Beijing Anzhen Hospital of Capital Medical University (Meng Zhang); and China-Japan Friendship Hospital (Kaiyuan Zhen and Zhu Zhang).

\section{External Reviewers}

General Hospital of Northern Theater Command (Lei Liu and Po Zhang); Peking University Third Hospital (Jing Zhang); Beijing Jishuitan Hospital (Yunjian Zhang); Beijing Hospital (Baomin Fang and Yongjun Li); the First Affiliated Hospital of Bengbu Medical College (Qixia Xu); Institute of Respiratory Diseases of Changsha Medical University (Aiguo Dai); the First Affiliated Hospital of Dalian Medical University (Zhonghe Zhang); the Second Affiliated Hospital of the Third Military Medical University (Ye Fan); Dongguan People's Hospital (Ruhong Xu); the First Affiliated Hospital of Fujian Medical University (Chaosheng Deng); Dongguan People's Hospital (Ping Zhang); State Key Laboratory of Respiratory Diseases of National Clinical Research Center for Respiratory Disease of Guangzhou Institute of Respiratory Health of the First Affiliated Hospital of Guangzhou Medical University (Cheng Hong, Jian Wang, and Nuofu Zhang); Guizhou Provincial People's Hospital (Weijia Liu); the First Affiliated Hospital of Henan University of Science and Technology (Yimin Mao); Key Laboratory of Respiratory Diseases of the Ministry of Health of the Department of Pathophysiology of Tongji Medical College of Huazhong University of Science and Technology (Qinghua Hu); Tongji Hospital Affiliated to Tongji Medical College of Huazhong University of Science and Technology (Guohua Zhen); the Second Hospital of Jilin University (Wei Xu); the Sixth Medical Center of PLA General Hospital (Yuying Li); Yan'an Hospital Affiliated to Kunming Medical University (Xiqian Xing); the First Affiliated Hospital with Nanjing Medical University (Weiping Xie); Inner Mongolia Baogang Hospital (Xiyuan Xu); Inner Mongolia People's Hospital (Jialie Wang); Shanxi Provincial People's Hospital (Aizhen Zhang); the First Hospital of Shanxi Medical University (Xiaoyun Hu and Yiwei Shi); Beijing Anzhen Hospital of Capital Medical University (Guangfa Zhu); Sichuan Provincial People's Hospital (Lu Guo); Tianjin Haihe Hospital (Qi Wu); Tianjin Medical University General Hospital (Jianlong Men and Lixia Dong); the First Affiliated Hospital of Wenzhou Medical University (Liangxing Wang); the First Affiliated Hospital of Xi'an Jiaotong University (Manxiang Li); Second People's Hospital of Tibet Autonomous Region (Wa Da); the People's Hospital of Xinjiang Uygur Autonomous Region (Ying Chen); Traditional Chinese Medicine Hospital of Xinjiang Uygur Autonomous Region (Huiqin Yang); the First Affiliated Hospital of Xinjiang Medical University (Hui Liu);
Tumor Hospital Affiliated to Xinjiang Medical University (Qin Luo); Sir Run Run Shaw Hospital Affiliated to School of Medicine of Zhejiang University (Ruifeng Zhang and Kejing Ying); the First Hospital of China Medical University (Fan Liu); and Fuwai Hospital of CAMS and PUMC (Zhihong Liu).

\section{Note}

The present consensus statement was based on its Chinese version which has been published previously in the National Medical Journal of China, 2020 March, 100 (11):808-813. DOI: $10.3760 /$ cma.j.cn112137-2020020900227, with full permission from the journal office and the above academic groups.

\section{Funding}

This guideline is funded by the Chinese Academy of Engineering emergency research and cultivation project for COVID-19 (grant number: 2020-KYGG-01-05), National Key Research and Development Program of China (grant numbers: 2016YFC0905600 and 2016YFC0901104), CAMS Innovation Fund for Medical Sciences (grant number: 2018I2M-1-003), and National Natural Science Foundation of China (grant numbers: 81570049 and 81970058).

Conflict of Interest

None declared.

\section{Acknowledgments}

Authors appreciate the continuous support and contributions from Profs. Benjamin Brenner, Ajay Kakkar, and Grégoire Le Gal. The authors also thank Dr. Xinwang Chen for providing technical and editorial support for the development of the manuscript. They would also thank all the expert group members from different area who participated in the review, discussion of the Statement, and all professionals who are battling on the frontline of the COVID-19 epidemic.

\section{References}

1 Huang C, Wang Y, Li X, et al. Clinical features of patients infected with 2019 novel coronavirus in Wuhan, China. Lancet 2020;395 (10223):497-506

2 Chen N, Zhou M, Dong X, et al. Epidemiological and clinical characteristics of 99 cases of 2019 novel coronavirus pneumonia in Wuhan, China: a descriptive study. Lancet 2020;395(10223):507-513

3 Tang N, Li D, Wang X, Sun Z. Abnormal coagulation parameters are associated with poor prognosis in patients with novel coronavirus pneumonia. J Thromb Haemost 2020;18(04):844-847

4 General Office of the National Health Commission. Diagnosis and treatment scheme of the novel coronavirus pneumonia (trial version 5). Available at: http://www.nhc.gov.cn/xcs/zhengcwj/ 202002/3b09b894ac9b4204a79db5b8912d4440.shtml. Accessed March 15, 2020

5 World Health Organization. Clinical management of severe acute respiratory infection (SARI) when novel coronavirus (2019-nCoV) infection is suspected (Interim guidance Version 1.2). Available at: https://www.who.int/publications-detail/clinical-management-ofsevere-acute-respiratory-infection-when-novel-coronavirus-(ncov)infection-is-suspected. Accessed March 15, 2020

6 World Health Organization. Clinical management of severe acute respiratory infection when Middle East respiratory syndrome 
coronavirus (MERS-CoV) infection is suspected (Interim guidance version 15.1). Updated January 2019. Available at: https://www. who.int/csr/disease/coronavirus_infections/case-management-ipc/en/. Accessed March 15, 2020

7 Pulmonary Embolism and Pulmonary Vascular Diseases Group of Chinese Thoracic Society, Pulmonary Embolism and Pulmonary Vascular Disease Working Committee of Chinese Association of Chest Physicians, National Cooperation Group on Prevention and Treatment of Pulmonary Embolism and Pulmonary Vascular Disease. Prevention and treatment of venous thromboembolism associated with COVID-19 infection: a consensus statement (in Chinese). Zhonghua Yi Xue Za Zhi 2020;100(11):808-813

8 Schünemann HJ, Cushman M, Burnett AE, et al. American Society of Hematology 2018 guidelines for management of venous thromboembolism: prophylaxis for hospitalized and nonhospitalized medical patients. Blood Adv 2018;2(22):3198-3225

9 Canadian Critical Care Society. Canadian Association of Emergency Physicians, Association of Medical Microbiology \& Infectious Diseases Canada. Ebola Clinical Care Guidelines. A guide for clinicians in Canada. Available at: https://caep.ca/wp-content/ uploads/2016/03/ebola_clinical_care_guideline_english_201505. pdf. Accessed March 15, 2020

10 Levy MM, Baylor MS, Bernard GR, et al; National Heart, Lung, and Blood Institute; Centers for Disease Control and Prevention; Institute of Allergy and Infectious Diseases. Clinical issues and research in respiratory failure from severe acute respiratory syndrome. Am J Respir Crit Care Med 2005;171(05):518-526

11 Expert Committee of Thrombosis and Blood Vessels of China Health Promotion Foundation, Pulmonary Embolism and Pulmonary Vascular Diseases Group of Chinese Thoracic Society, Pulmonary Embolism and Pulmonary Vascular Disease Working Committee of Chinese Association of Chest Physicians. Prophylaxis and management of venous thromboembolism in hospitalized patients. (in Chinese). Zhonghua Yi Xue Za Zhi 2018;98(18): 1383-1388

12 Pulmonary Embolism and Pulmonary Vascular Diseases Group of Chinese Thoracic Society, Pulmonary Embolism and Pulmonary Vascular Disease Working Committee of Chinese Association of Chest Physicians, National Cooperation Group on Prevention and Treatment of Pulmonary Embolism and Pulmonary Vascular Disease. Guidelines for the diagnosis, treatment and prevention of pulmonary thromboembolism. (in Chinese). Zhonghua Yi Xue Za Zhi 2018;98(14):1060-1087

13 Guidelines for the Prevention and Treatment of Thrombotic Diseases in China issued by expert committee. Guidelines for the Prevention and Treatment of Thrombotic Diseases in China. (in Chinese). Zhonghua Yi Xue Za Zhi 2018;98(36):2861-2888

14 Konstantinides SV, Meyer G, Becattini C, et al; ESC Scientific Document Group. 2019 ESC Guidelines for the diagnosis and management of acute pulmonary embolism developed in collaboration with the European Respiratory Society (ERS). Eur Heart J 2020;41(04):543-603

15 Brouwers MC, Kho ME, Browman GP, et al; AGREE Next Steps Consortium. AGREE II: advancing guideline development, reporting and evaluation in health care. CMAJ 2010;182(18):E839-E842

16 Chen Y, Yang K, Marušic A, et al; RIGHT (Reporting Items for Practice Guidelines in Healthcare) Working Group. A tool for practice guidelines in health care: the RIGHT statement. Ann Intern Med 2017;166(02):128-132

17 Barbar S, Noventa F, Rossetto V, et al. A risk assessment model for the identification of hospitalized medical patients at risk for venous thromboembolism: the Padua Prediction Score. J Thromb Haemost 2010;8(11):2450-2457

18 Cronin M, Dengler N, Krauss ES, et al. Completion of the updated Caprini risk assessment model (2013 version). Clin Appl Thromb Hemost 2019;25:1076029619838052

19 Chen H, Guo J, Wang C, et al. Clinical characteristics and intrauterine vertical transmission potential of COVID-19 infection in nine pregnant women: a retrospective review of medical records. Lancet 2020;395(10226):809-815

20 Leffert L, Butwick A, Carvalho B, et al; members of the SOAP VTE Taskforce. The Society for Obstetric Anesthesia and Perinatology Consensus Statement on the anesthetic management of pregnant and postpartum women receiving thromboprophylaxis or higher dose anticoagulants. Anesth Analg 2018;126(03):928944

21 Dellinger RP, Levy MM, Rhodes A, et al; Surviving Sepsis Campaign Guidelines Committee including The Pediatric Subgroup. Surviving Sepsis Campaign: international guidelines for management of severe sepsis and septic shock, 2012. Intensive Care Med 2013;39 (02):165-228

22 Duranteau J, Taccone FS, Verhamme P, Ageno W; ESA VTE Guidelines Task Force. European guidelines on perioperative venous thromboembolism prophylaxis: Intensive care. Eur J Anaesthesiol 2018;35(02):142-146

23 Committee of Critical Care Medicine, Chinese Association of Chest Physician, Chinese Medical Doctor Association; Critical Care Medicine Group, Chinese Thoracic Society, Chinese Medical Association. [Recommendations for clinical application of extracorporeal membrane oxygenation in adults severe acute respiratory failure]. Zhonghua Jie He He Hu Xi Za Zhi 2019;42(09): 660-684

24 Cuker A, Arepally GM, Chong BH, et al. American Society of Hematology 2018 guidelines for management of venous thromboembolism: heparin-induced thrombocytopenia. Blood Adv 2018;2(22):3360-3392

25 Selleng S, Selleng K. Heparin-induced thrombocytopenia in cardiac surgery and critically ill patients. Thromb Haemost 2016;116 (05):843-851

26 Barlow A, Barlow B, Reinaker T, Harris J. Potential role of direct oral anticoagulants in the management of heparin-induced thrombocytopenia. Pharmacotherapy 2019;39(08):837-853

27 Thomas O, Lybeck E, Strandberg K, Tynngård N, Schött U. Monitoring low molecular weight heparins at therapeutic levels: doseresponses of, and correlations and differences between aPTT, anti-factor Xa and thrombin generation assays. PLoS One 2015; 10(01):e0116835

28 Crowther M, Lim W. Use of low molecular weight heparins in patients with renal failure; Time to re-evaluate our preconceptions. J Gen Intern Med 2016;31(02):147-148

29 Helfer H, Siguret V, Mahé I. Tinzaparin sodium pharmacokinetics in patients with chronic kidney disease: practical implications. Am J Cardiovasc Drugs 2019. Doi: 10.1007/s40256-019-00382-0

30 Hughes S, Szeki I, Nash MJ, Thachil J. Anticoagulation in chronic kidney disease patients-the practical aspects. Clin Kidney J 2014; 7(05):442-449

31 Minet C, Potton L, Bonadona A, et al. Venous thromboembolism in the ICU: main characteristics, diagnosis and thromboprophylaxis. Crit Care 2015;19(01):287

32 Arabi YM, Al-Hameed F, Burns KEA, et al; Saudi Critical Care Trials Group. Adjunctive intermittent pneumatic compression for venous thromboprophylaxis. N Engl J Med 2019;380(14):1305-1315

33 Tang N, Bai H, Chen X, Gong J, Li D, Sun Z. Anticoagulant treatment is associated with decreased mortality in severe coronavirus disease 2019 patients with coagulopathy. J Thromb Haemost 2020;18(5):1094-1099

34 Han H, Yang L, Liu R, et al. Prominent changes in blood coagulation of patients with SARS-CoV-2 infection. Clin Chem Lab Med 2020. Doi: 10.1515/cclm-2020-0188

35 Zhou F, Yu T, Du R, et al. Clinical course and risk factors for mortality of adult inpatients with COVID-19 in Wuhan, China: a retrospective cohort study. Lancet 2020;395(10229):1054-1062

$36 \mathrm{Wu} \mathrm{C}$, Chen X, Cai Y, et al. Risk factors associated with acute respiratory distress syndrome and death in patients with coronavirus disease 2019 pneumonia in Wuhan, China. JAMA Intern Med 2020. Doi: 10.1001/jamainternmed.2020.0994 
948 Prevention and Treatment of VTE Associated with COVID-19 Zhai et al.

37 Cao B, Wang Y, Wen D, et al. A trial of lopinavir-ritonavir in adults hospitalized with severe Covid-19. N Engl J Med 2020;382 (19):1787-1799

38 Baden LR, Rubin EJ. Covid-19 - The search for effective therapy. N Engl J Med 2020;382(19):1851-1852
39 Driggin E, Madhavan MV, Bikdeli B, et al. Cardiovascular considerations for patients, health care workers, and health systems during the coronavirus disease 2019 (COVID-19) pandemic. J Am Coll Cardiol 2020;75(18):2352-2371 